\title{
Galaxies around the giant double radio source DA 240
}

\section{Redshifts and the discovery of an unusual association}

\author{
B. Peng ${ }^{1}$, R. G. Strom ${ }^{2,3, \star}$ J. Wei ${ }^{1, \star \star}$, and Y. H. Zhao ${ }^{1, \star \star \star}$ \\ 1 National Astronomical Observatories, Chinese Academy of Sciences, Beijing 100012, PR China \\ 2 ASTRON, Postbus 2, 7990 AA Dwingeloo, The Netherlands \\ 3 Astronomical Institute, University of Amsterdam, The Netherlands
}

Received 19 September 2003 / Accepted 30 October 2003

\begin{abstract}
We have investigated galaxies near the giant radio source DA 240 in a study of the source's environment. Spectroscopic observations were obtained with the $2.16 \mathrm{~m}$ optical telescope of the National Astronomical Observatories of China. We have measured the redshifts of 26 objects, and find that 16 have nearly the same value as the DA 240 galaxy, and hence form a substantial group. Most of the others have lower redshifts and are probably outlying members of the Zwicky cluster $\mathrm{Zw} 0756.1+5616$. We note that the radio galaxy host dominates the group. Ten of the galaxies in the group are aligned with the lobes of the radio source, and eight are projected on the components. We suggest that there may be a causal relationship, and find some evidence for enhanced activity among these galaxies. Moreover, there is a gradient in their velocities along the source axis. Whatever its explanation, we suggest that it establishes a dynamic timescale of some $10^{9} \mathrm{yr}$. This may have been the time since the last merger, which triggered the giant radio source. The individual galaxy spectra are also presented and briefly discussed.
\end{abstract}

Key words. galaxies: active - galaxies: individual: DA 240 - galaxies: ISM - galaxies: distances and redshifts - techniques: spectroscopic - ISM: jets and outflows

\section{Introduction}

Giant radio galaxies (GRGs) are defined as having an overall projected size $\geq 1 \mathrm{Mpc}$ (e.g. Klein et al. 1994, for $H_{0}=$ $75 \mathrm{~km} \mathrm{~s}^{-1} \mathrm{Mpc}^{-1}$ ). As a class, they constitute less than $10 \%$ of flux-limited radio source samples (Laing et al. 1983). Among the numerous studies of individual GRGs, there have been those by Willis \& Strom (1978), Willis et al. (1978), Bridle et al. (1979), Strom \& Willis (1980), Barthel et al. (1985), Jägers (1986, 1987a and 1987b) at the lower frequencies; and by Baker et al. (1974), Stoffel \& Wielebinski (1978), Strom et al. (1981), Klein et al. (1994) and Saripalli et al. (1996) at higher frequencies. There has also been a search for new candidates based on the WENSS survey (Schoenmakers et al. 2001). GRGs are often of intermediate radio luminosity with values in the transition region between FR I and II types (Fanaroff \& Riley 1974), and are believed to be advancing outwards through the low-density $\left(10^{-5}-10^{-6} \mathrm{~cm}^{-3}\right)$ intergalactic medium (IGM), with estimated spectral ages of about $10^{7}-10^{8}$ years (Mack et al. 1998). DA 240, the subject of this investigation, lies just at the FR I/II border both in terms of its luminosity, and morphologically since its NE lobe contains

\footnotetext{
Send offprint requests to: B. Peng, e-mail: pb@bao.ac.cn

* e-mail: strom@astron.nl

$\star \star$ e-mail: wjy@bao.ac.cn

$\star \star \star$ e-mail: yzhao@lamost.org
}

a prominent hotspot, but one significantly set back from the outer edge of the lobe.

DA 240 was among the first GRGs to be recognized as such, given its angular size (some $40^{\prime}$ arc) and redshift, $z=$ 0.0356 (Willis et al. 1974). The most prominent radio feature the hotspot in the eastern lobe (catalogued as the radio source 4C 56.16) - was originally thought to be emission from the $z=0.0352$ galaxy VV 9-13-66. It is, however, quite separate from 4C 56.16, a radio source in its own right, and a companion to the DA 240 galaxy (VV 9-13-57). Van Breugel et al. (1983) found several foreground $(z \simeq 0.02)$ galaxies, one of which overlaps the DA 240 one, and which they designate as the low velocity system (LVS). These may all belong to the Zwicky cluster Zw 0756.1+5616 (Zwicky \& Herzog 1966). DA 240 and the galaxies around it lie on the western edge of Zw $0756.1+5616$, and may even be loosely connected with it.

In an effort to clarify the nature of the galaxies surrounding DA 240, we have tried to obtain spectra of all those brighter than $m_{r} \simeq 17$, over 26 objects, using the $2.16 \mathrm{~m}$ optical telescope in Beijing (Xinglong Station of the NAOC). We have also reanalysed WSRT observations of DA 240 made at $49 \mathrm{~cm}$, for comparison with the optical galaxies. In the following section, we introduce the radio and optical observations and related data reduction. The optical spectra of candidate galaxies are also illustrated. In Sect. 3 we present the high dynamic range total intensity radio map, and discuss some possible interpretations 
of our observational findings. Finally concluding remarks are made in Sect. 4.

Throughout this paper the radio spectral index is defined by $S_{v} \propto v^{-\alpha}$, the deceleration parameter $q_{0}=0.5$ and a Hubble constant of $H_{0}=50 \mathrm{~km} \mathrm{~s}^{-1} \mathrm{Mpc}^{-1}$ are used.

\section{Observations and data reduction}

\subsection{Sample selection}

Using the Digital Sky Survey (DSS), we have searched for galaxies within a region of about $1^{\circ}$ centred on the DA $240 \mathrm{nu}-$ cleus, and find some 26 candidates (brighter than $\sim 17^{\mathrm{m}}$ in the $R$ band), as listed in Table 1 . We believe this list to be complete for nonstellar objects within the search region for $m<17^{\mathrm{m}}$ on the red DSS. Each source is numbered, in order of right ascension (RA), and there follow the source coordinates in J2000 (RA and Dec), apparent $R$ band magnitude (taken from the USNO-A2.0 catalogue, which used POSS-I data; the magnitudes are based on a stellar scale, and no correction has been attempted), and the redshift ( $z$ ) if known, in Cols. 2-5 respectively. It should be noted that four more galaxies (source numbers 29-32 in Table 1) would have been observed if we had included objects one magnitude fainter.

In our sample the galaxy ARK141 (=VV 9-13-66; source number 21) is included in the NASA/IPAC Extragalactic Database (NED) at roughly the same redshift as the DA 240 nucleus (i.e., CGCG262-029 = VV 9-13-57). It, VV 9-13-57, as well as three others, SBS0745+557 at $z \simeq 0.0040$, CGCG262035 at $z \simeq 0.0199$, and object No. $6(z=0.0340$ from Rines et al. 2000), had known redshifts before we began. To check on our data quality these five galaxies were re-observed by us. The previously known redshifts are given in the table. Finally we have included another 3 galaxies: the DA 240 LVS at $z \simeq 0.024$ (Van Breugel et al. 1983), UGC 04035 at $z \simeq 0.0193$ (from NED), and No. 11 (from De Bruyn, private communication) in Table 1. For the first two, there is no magnitude entry.

The distribution of all 32 candidate galaxies on the sky is shown in Fig. 1, which includes the 4 galaxies one magnitude fainter than the original 26. (We plan to follow them up in the future.) It is noticeable that there is a tendency for many of them to concentrate along the radio axis of DA 240 (see Sect. 3 and Fig. 4), although there are clearly others off to the south in particular, which is in the direction of the central concentration of the cluster $\mathrm{Zw} 0756.1+5616$. The first crucial test of an association is, of course, whether the galaxies all have the same redshift. We therefore requested observing time to obtain spectra of them. The spectra would also enable us to identify candidate AGNs, to see if the others are similar to VV 9-13-66 and whether the galaxies which may be immersed within the radio lobes are actually affected by the presence of a greater cosmic-ray background.

\subsection{Optical data}

In order to determine redshifts of the selected objects, spectroscopic observations have been made with the $2.16 \mathrm{~m}$ telescope and OMR spectrograph of Xinglong Station, NAOC, using a
Table 1. 26 selected candidate galaxies with magnitude brighter than $\simeq 17^{\mathrm{m}}$ in the $R$ band, and two additional ones from other sources (no magnitude known). Eight galaxies have previously measured $z$, including one from A. G. de Bruyn (indicated by an asterisk). The last four galaxies listed are those one magnitude fainter than the sample of 26.

\begin{tabular}{|c|c|c|c|c|}
\hline \multirow[t]{2}{*}{ No. } & RA (J2000) & Dec. (J2000) & \multirow{2}{*}{$\begin{array}{c}R \\
\text { app. mag. }\end{array}$} & \multirow{2}{*}{$\begin{array}{l}\text { Redshift } \\
z\end{array}$} \\
\hline & $\mathrm{h} \quad \mathrm{m} \quad \mathrm{s}$ & $\circ \quad, \quad \prime$ & & \\
\hline 1 & 074603.94 & 560536.2 & 13.1 & \\
\hline 2 & 074620.83 & 561157.8 & 13.2 & \\
\hline 3 & 074630.26 & 553844.2 & 14.6 & \\
\hline 4 & 074651.07 & 561042.1 & 14.2 & \\
\hline 5 & 074732.90 & 554148.2 & 16.5 & \\
\hline 6 & 074735.52 & 554118.5 & 12.6 & 0.0340 \\
\hline 7 & 074745.94 & 554641.4 & 17.2 & \\
\hline 8 & 074751.26 & 554706.7 & 15.7 & \\
\hline 9 & 074755.12 & 554628.3 & 13.9 & \\
\hline 10 & 074757.75 & 554510.1 & 15.0 & \\
\hline 11 & 074808.29 & 554815.5 & 14.1 & $0.026^{*}$ \\
\hline 12 & 074836.87 & 554858.3 & 10.7 & 0.0356 \\
\hline 13 & 074836.87 & 554858.3 & & 0.024 \\
\hline 14 & 074844.76 & 560035.5 & 16.8 & \\
\hline 15 & 074901.27 & 552532.0 & 13.9 & \\
\hline 16 & 074909.84 & 553616.8 & 11.4 & 0.0040 \\
\hline 17 & 074912.28 & 554312.2 & 14.2 & \\
\hline 18 & 074912.62 & 555233.6 & 14.7 & \\
\hline 19 & 074924.56 & 555813.4 & 14.1 & \\
\hline 20 & 074937.90 & 555244.4 & 14.3 & \\
\hline 21 & 074958.51 & 555528.0 & 12.7 & 0.0352 \\
\hline 22 & 075007.12 & 553514.5 & 13.2 & \\
\hline 23 & 075008.40 & 552303.0 & & 0.0193 \\
\hline 24 & 075016.36 & 552429.9 & 11.0 & 0.0199 \\
\hline 25 & 075019.34 & 554041.5 & 15.0 & \\
\hline 26 & 075021.33 & 555401.4 & 11.7 & \\
\hline 27 & 075028.99 & 552901.8 & 13.0 & \\
\hline 28 & 075053.10 & 552758.1 & 12.8 & \\
\hline 29 & 074707.51 & 554345.5 & & \\
\hline 30 & 074733.70 & 554509.0 & & \\
\hline 31 & 074913.49 & 555648.5 & & \\
\hline 32 & 074917.14 & 555303.1 & & \\
\hline
\end{tabular}

Tektronix $1024 \times 1024 \mathrm{CCD}$ as detector. We used a grism of $300 \mathrm{~g} \mathrm{~mm}^{-1}$ to get a wavelength coverage of $3800-8000 \AA$, and a $2^{\prime \prime}$ wide slit, providing an average spectral resolution of $9.6 \AA$ as measured from the comparison spectra. Exposures of 1500$5400 \mathrm{~s}$ were taken on the nights of January 16 and 17 in 2001, and 3 nights during February $4-6$ in 2002. The wavelength calibration was carried out using helium and argon lamps. The flux calibration was provided by exposures of KPNO standard stars (Massey et al. 1988), such as Feige 34, G191B2B, etc. The atmospheric extinction was corrected by using the mean extinction of Xinglong. The telluric $\mathrm{O}_{2}$ aborption bands at 


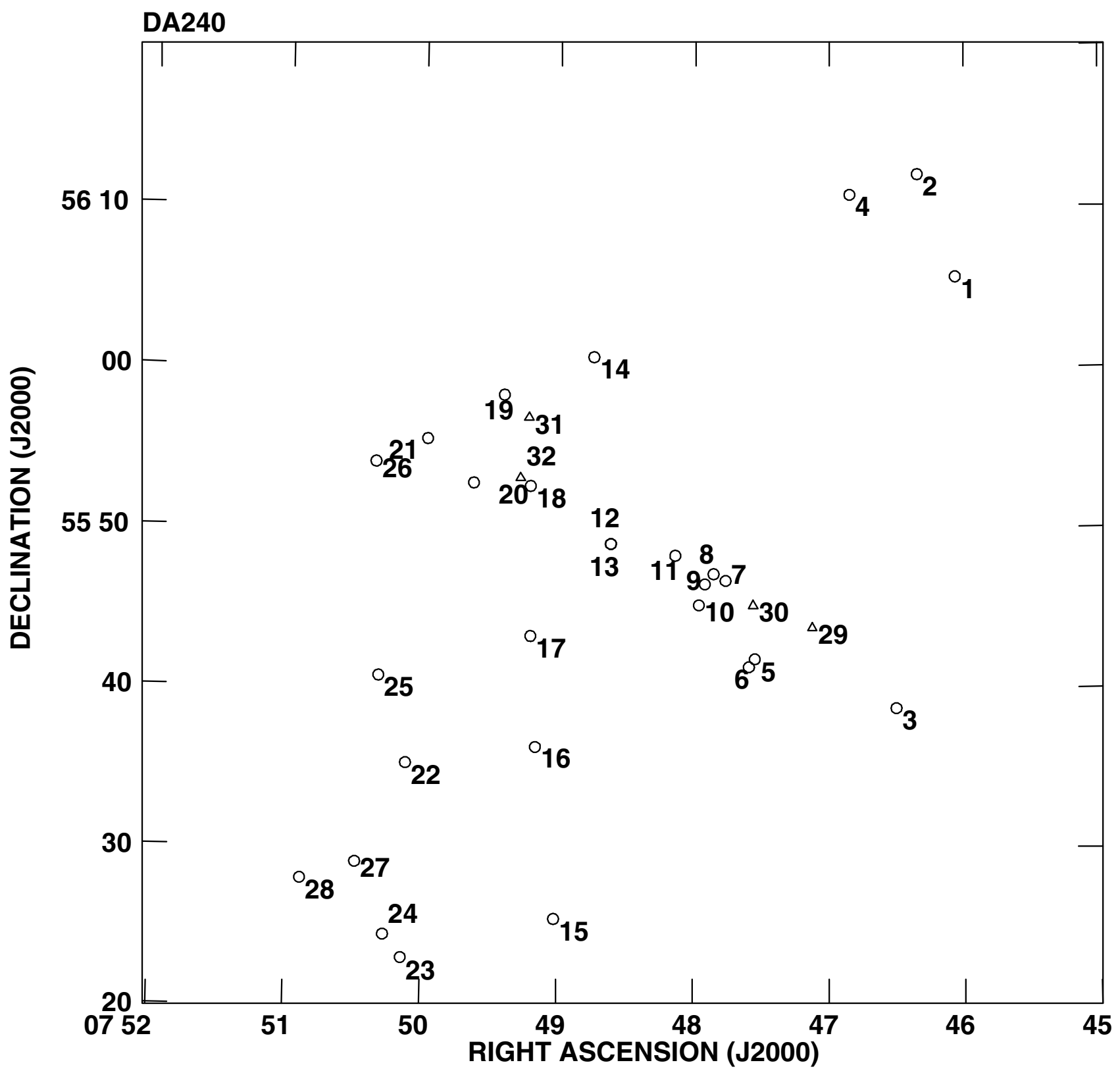

Fig. 1. A chart showing the locations of the 32 galaxies listed in Table 1, numbered as in the table. Triangles indicate the four additional candidates, Nos. 29, 30, 31 and 32, also aligned with the lobes of DA 240, which would have been included if we had selected objects one magnitude fainter.

$6870 \AA$ and $7620 \AA$ A were not removed. The data reduction and calibration were performed using IRAF software. After editing and wavelength calibration, each spectrum was inspected for emission and absorption features. Candidate lines were fitted to determine their wavelength using GAUSSFIT, the redshift $(z)$ was calculated after line identification, and a (weighted) average $z$ was determined. The error was computed from the absolute calibration uncertainty, a noise estimate, and the internal consistency of multiple lines, all combined in quadrature. The reduced 1D spectra are shown in Fig. 2, in order of RA.

The redshifts we obtained (heliocentric values) from the absorption and/or emission lines are listed in the fifth column of Table 2, where the same numbers used for our targets in Table 1 are given in the first column, the identified group types in the second column refer to galaxies having redshifts almost the same as the DA 240 nucleus (group A), a trio with $z \simeq 0.025$ (including the LVS; group A1), those with $z \simeq 0.019$ (B) and the rest $(\mathrm{R})$. The exposure time for each observation is listed in the sixth column, and some notes about the source spectra appear in the seventh column. We note that for one of the galaxies - SBS0745+557 (No. 16) - our redshift $(z=0.0183)$ is very different from that listed by NED (0.003996). We have rechecked our determination very carefully, and are quite confident of its correctness. We can only conclude that the NED value, which comes from the UZC (Falco et al. 1999), is in error.

Rough classifications following the criteria of Veilleux \& Osterbrock (1987) were made for each object observed by us. 

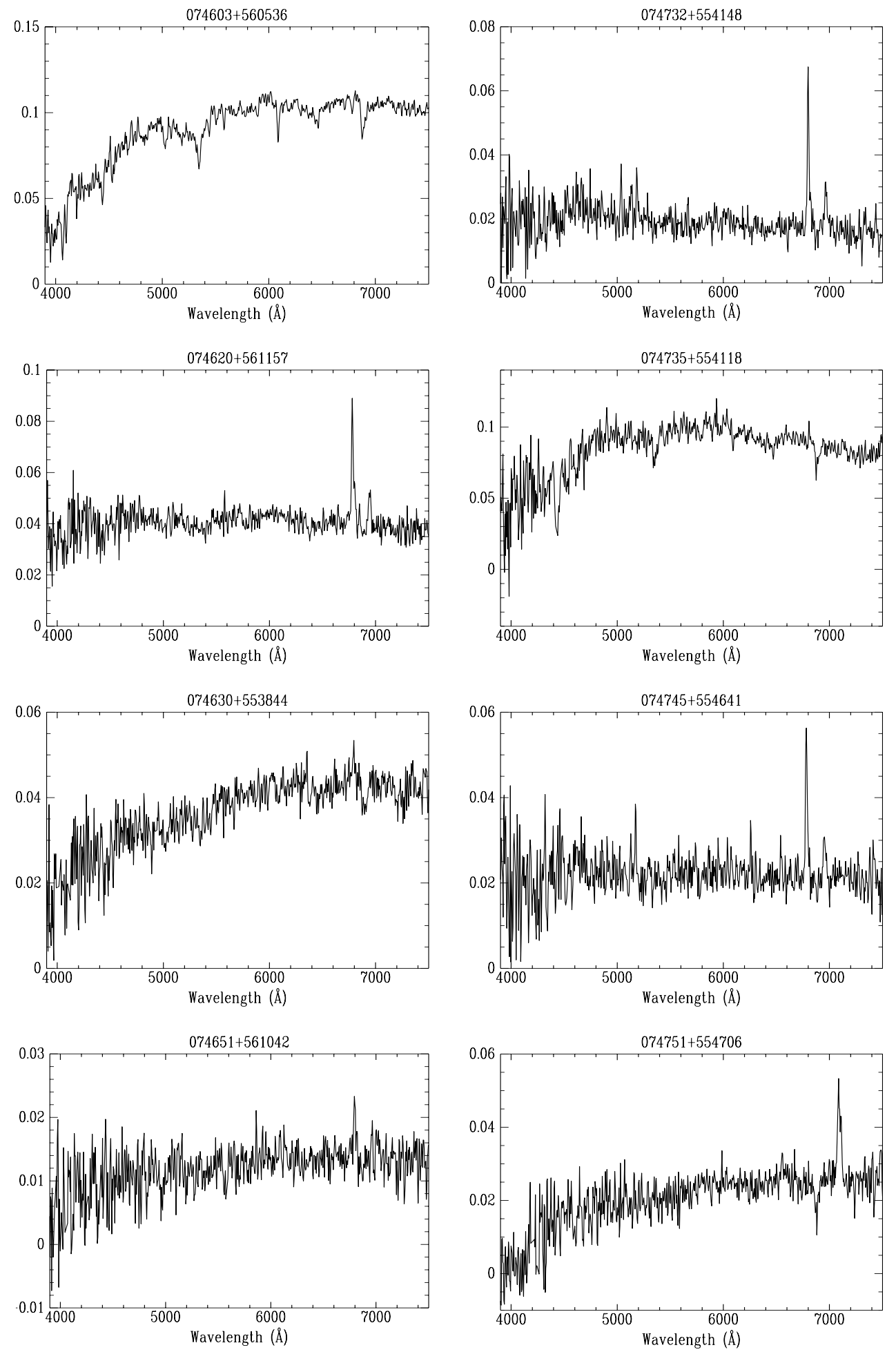

Fig. 2. Redshift un-corrected spectra of the selected objects around the giant radio source DA 240, observed with the $2.16 \mathrm{~m}$ optical telescope at Xinglong Station of the NAOC. The vertical axis is in units of $1 \times 10^{-14}$ counts, and the horizontal axis is in units of $10^{-10} \mathrm{~m}$.

Galaxies without emission lines are classified as normal (G). Objects with $\mathrm{H} \alpha$ but weak [NII] $\lambda 6583$ and [OIII] $\lambda 5007$ emission lines are classified as HII, while objects with a strong [NII] $\lambda 6583$ emission line are classified as Seyfert 2 (S2) or LINERs. However, we could not further distinguish between Seyfert $2 \mathrm{~s}$ and LINERs for most of the objects since we could not get a good estimate of the line ratio between $\mathrm{H} \beta$ and [OIII] given the low signal to noise ratios of their spectra (hence the designation $\mathrm{S} 2 / \mathrm{L}$ ). Objects with $\mathrm{H} \alpha$ emission but with a low signal to noise ratio are classified simply as emission line galaxies (ELG). These type classifications are listed in the eighth column of Table 2. 

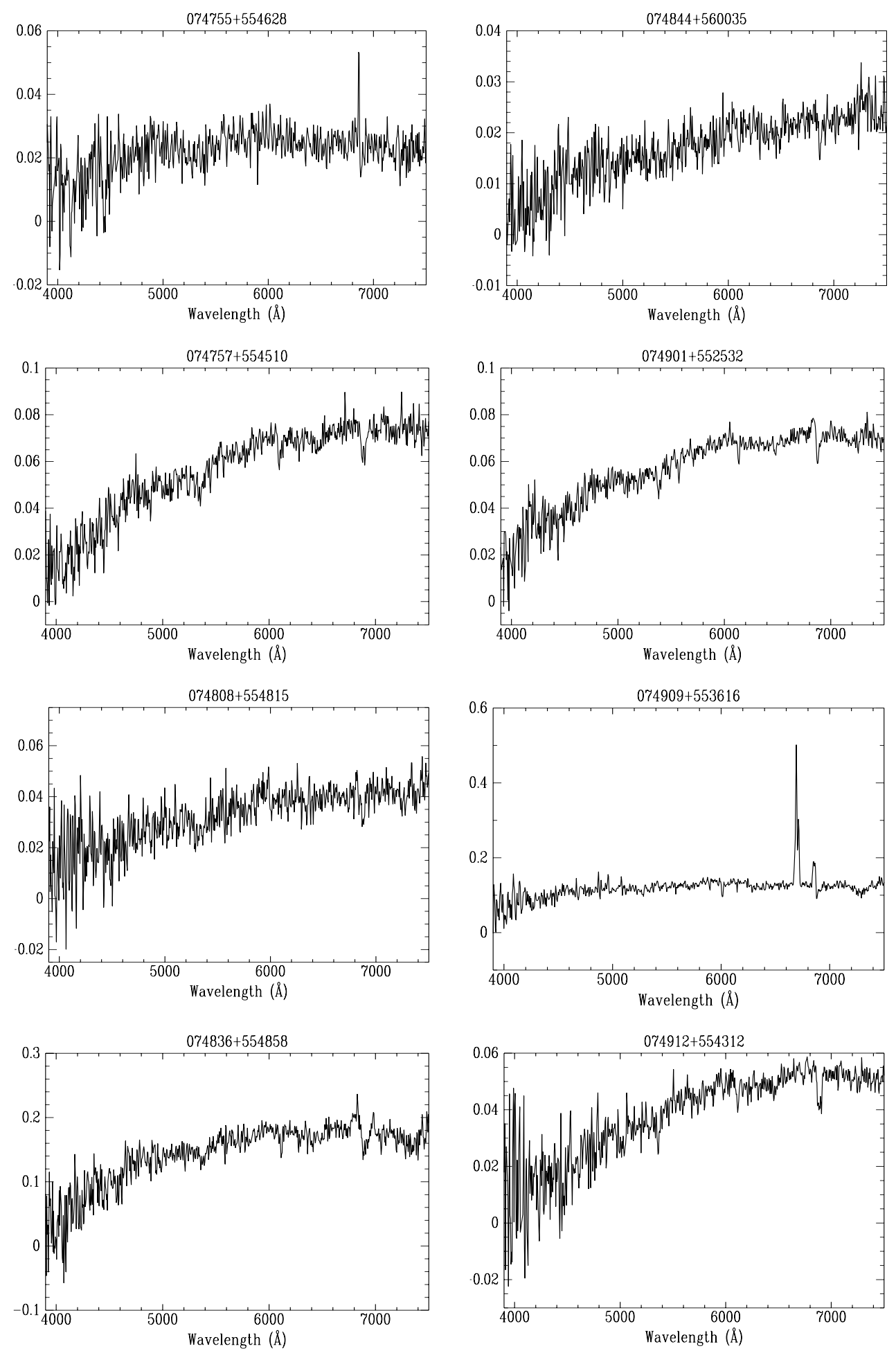

Fig. 2. continued.

\subsection{Radio data}

baselines as an aid to self-calibration (Noordam \& De Bruyn 1990). Standard analysis was done using the WSRT reduction We have reanalyzed existing WSRT $49 \mathrm{~cm}$ data for comparison with the optical galaxies. The data consisted of two 12 hour observations with 36 and $72 \mathrm{~m}$ settings of the shortest baseline, to obtain a synthesis map with a grating ring radius of $48^{\prime}$ arc (Högbom \& Brouw 1974). The data include redundant package NEWSTAR, and the final maps were CLEANed and restored with a $29^{\prime \prime} \times 35^{\prime \prime}$ Gaussian beam. The flux density scale is that of Baars et al. (1977). The map is presented in the following section. 

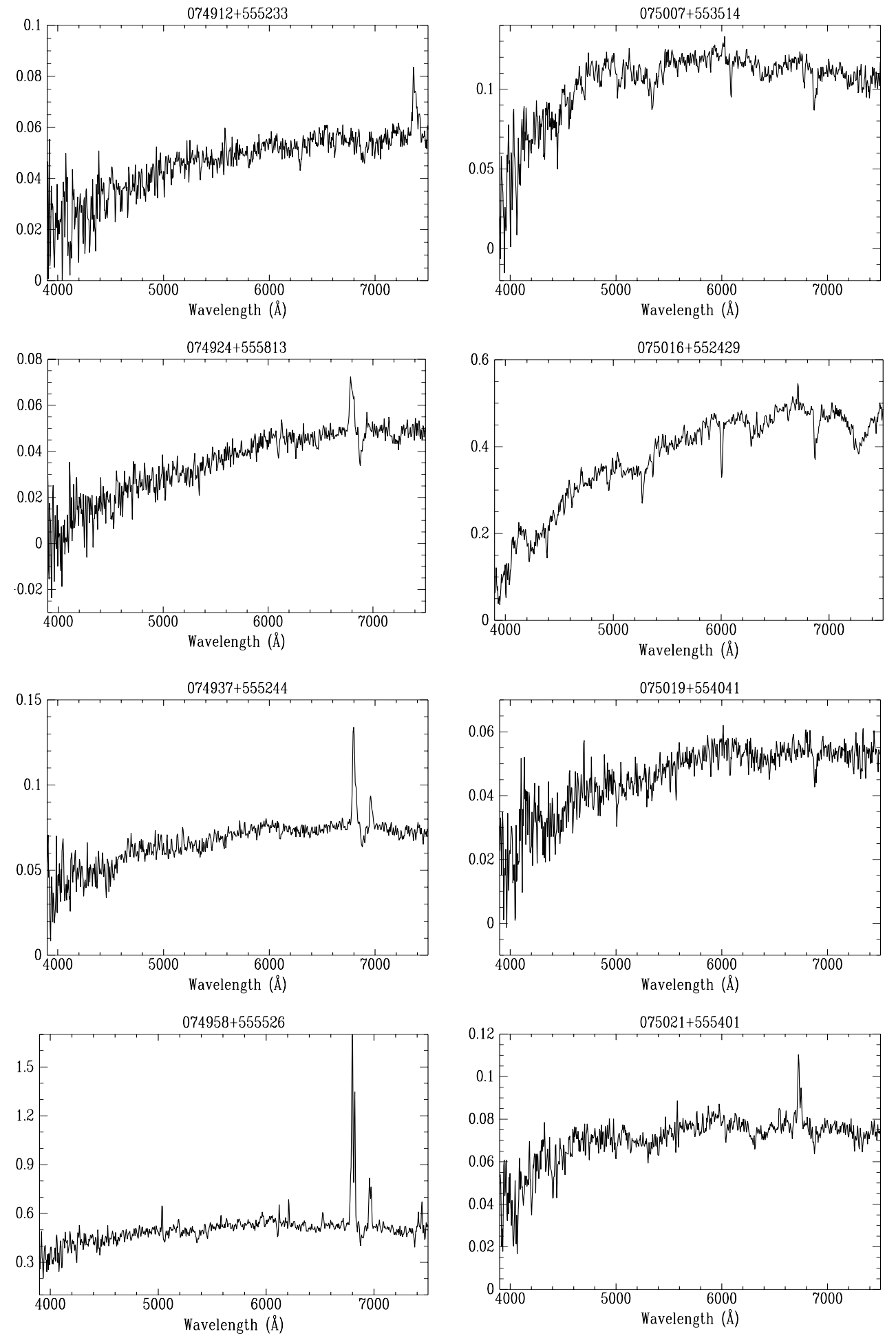

Fig. 2. continued.

\section{Results and discussion}

\subsection{Observational findings}

We have determined the redshifts of 26 galaxies within $0.5^{\circ}$ of DA 240, of which 21 have not been published before. They are all listed in Table 2, along with the redshifts of two galaxies from other sources. Most of these galaxies appear to belong to the DA 240 group, as can be best seen in a histogram

showing the distribution of all 28 with $z$ (Fig. 3). The main group with $0.032 \leq z \leq 0.038$ is readily apparent (the group A in Table 2, i.e., source numbers $1-7,9,10,12,14,17,19-22$, and 25). The galaxies at $z=0.0288$ (source 11) and $z=0.0416$ (source 15) may also belong, although their inclusion nearly doubles the dispersion of the ensemble. Moreover, on the basis of the $3 \sigma$ rejection criterion suggested by Yahil and Vidal (1977), both would be excluded, so we will leave them out. 

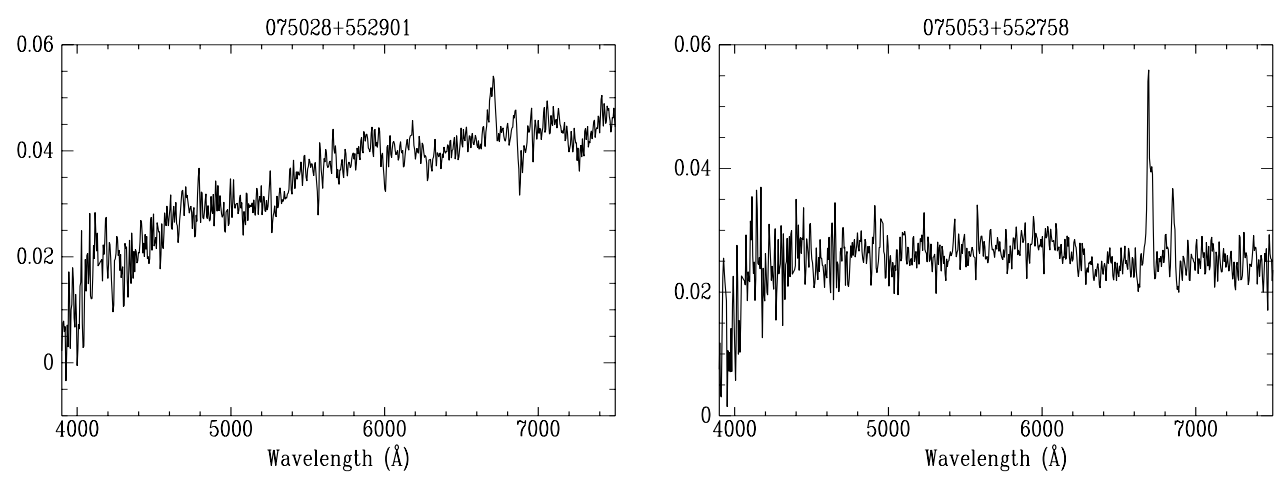

Fig. 2. continued.

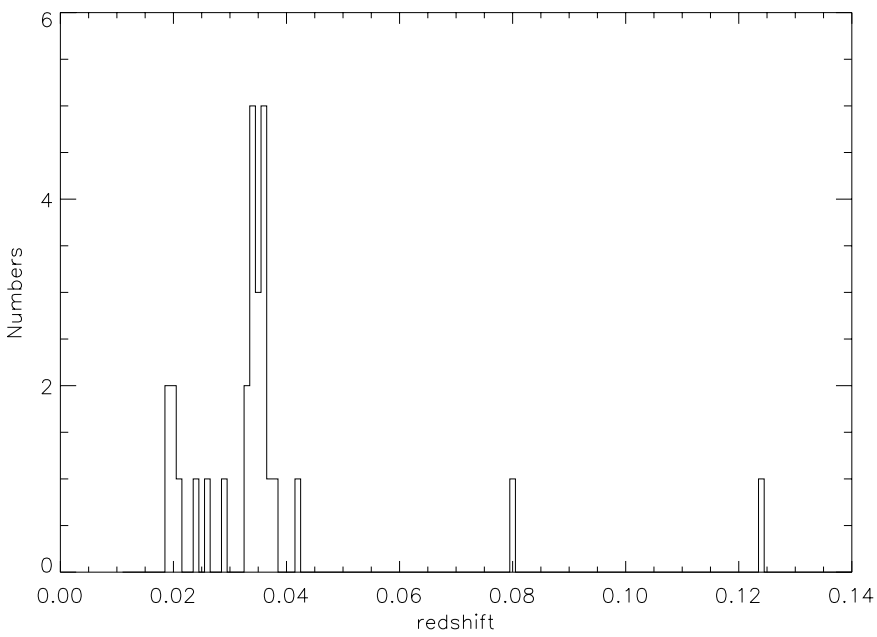

Fig. 3. Histogram of the redshift distribution for the 28 galaxies from Table 2.

The five galaxies with $z \simeq 0.019$, which form a small group (B in Table 2) to the southeast of DA 240, may indicate the edge of $\mathrm{Zw} 0756.1+5616$. The three intermediate $(z \simeq 0.025)$ galaxies (A1), which include the DA 240 LVS nearly coincident with VV 9-13-57, apparently lie between the two groups. The two galaxies with $z>0.07$ are presumably just background objects.

The locations of the galaxies of the groups A, A1 and B in Table 2 are shown, together with the $49 \mathrm{~cm}$ radio brightness distribution, in Fig. 4. It is clear that of the 17 galaxies in the DA 240 group, 11 lie along the major axis of the radio source, i.e., three in the eastern lobe, five in the western lobe, the central galaxy (VV 9-13-57), one a bit north of the eastern lobe, and one just beyond the western "tongue" of emission. In addition, there are two other candidate galaxies in each lobe (as shown in Fig. 1, source numbers 29-32) for which, however, we do not yet have redshift information. (Preliminary analysis of a spectrum recently obtained for source 31 suggests that it too belongs to group A.) The remaining 6 galaxies lie in two groups northwest and southeast of the radio axis. It is interesting to note that several of the DA 240 group galaxies are, like VV 9-13-57 itself, also radio sources, though weak. Of course this was already known of VV 9-13-66 near the bright eastern hotspot. All of those with detectable radio emission are seen projected against, or on the edge of, the radio lobes, suggesting a causal relationship.
Of course the alignment of over half the galaxies in the group with the radio lobes could be a chance coincidence, but a simple estimate (based on the area subtended by the lobes) suggests that the probability is much less than $10^{-2}$. An additional test which the system must pass is whether the kinematic lifetime of the galaxy group is greater than the age of the radio source. The velocity dispersions (corrected for the fact that we only measure the line-of-sight velocity) for the two groups coincident with each lobe are, $\sigma=500 \mathrm{~km} \mathrm{~s}^{-1}$ (eastern) and $\sigma=565 \mathrm{~km} \mathrm{~s}^{-1}$ (western). The lobe radii are both $\simeq 400 \mathrm{kpc}$, giving kinematic lifetimes of about $8 \times 10^{8} \mathrm{yr}(\mathrm{E})$, and $7 \times 10^{8} \mathrm{yr}(\mathrm{W})$. For the radio emission, the lifetime of a relativistic electron at an observed frequency, $v_{0}$, as a result of synchrotron or inverse-Compton losses is given by,

$\tau=\left[\frac{5 \times 10^{8}}{v_{0}(\mathrm{~Hz}) B^{3}(\mathrm{G})}\right]^{\frac{1}{2}} \mathrm{yr}$

from Strom et al. (1981). $B$ is either the magnetic field strength or, if inverse-Compton losses dominate, it is the magnetic field equivalent of the microwave background energy density. Using such formulae, and modelling the overall radio spectrum, Mack et al. (1998) conclude that the age of the radio emission is, $t \leq 10^{7} \mathrm{yr}$. The kinematic lifetime of the galaxy groups is thus comfortably larger than the radiation age of the lobes.

\subsection{Radio emission}

One of the surprising aspects of finding galaxies in the lobes of DA 240 is that one expects galaxies to appear in regions of higher than average density. However, we know from polarization measurements that the lobes have a quite low average density (Mack et al. 1997). They are strongly polarized at all wavelengths, even $90 \mathrm{~cm}$ where Faraday depolarization will be greatest. Mack et al. (1998) have analyzed data over a wide range of wavelengths, and conclude that the magnetic field strength must be, $B \simeq 0.5 \mu \mathrm{G}$, or $B \simeq B_{\mathrm{eq}}$, a result which has been found in other GRGs (Tsakiris et al. 1996). Consequently, the density of free electrons is, $n_{\mathrm{e}} \simeq 10^{-5} \mathrm{~cm}^{-3}$. This is surprising, though of course the much higher density in the galaxies themselves will have a minimal effect upon the depolarization of the lobes because of their small filling factor.

Both lobes have hotspots, but they are very asymmetric, with the eastern one some 50 times brighter than its 
Table 2. Summary of the observing results: 26 selected objects observed with the $2.16 \mathrm{~m}$ optical telescope, and two addtional ones from other sources like NED (observing dates left blank, $z$ enclosed in brackets).

\begin{tabular}{|c|c|c|c|c|c|c|c|}
\hline No. & G & RA+Dec name & $\begin{array}{c}\text { Obs. dates } \\
\text { yyyy.mm.dd }\end{array}$ & $\begin{array}{l}\text { Redshift } \\
z\end{array}$ & $\begin{array}{c}\text { Exposure } \\
\mathrm{s}\end{array}$ & Notes & $\mathrm{C}$ \\
\hline 1 & A & $074603+560536$ & 2002.02.04 & $0.0335 \pm 0.0004$ & 3600 & $\mathrm{H}_{\alpha}, \mathrm{H}_{\beta}, \mathrm{NaD}, \mathrm{Mg}^{\prime}$ "b", $G$ band & $\mathrm{G}$ \\
\hline 2 & A & $074620+561157$ & 2002.02 .05 & $0.0332 \pm 0.0005$ & 4800 & $\mathrm{H}_{\alpha},[\mathrm{SII}],[\mathrm{OI}]$ & HII \\
\hline 3 & A & $074630+553844$ & 2002.02 .05 & $0.0323 \pm 0.0005$ & 4200 & $\mathrm{H}_{\alpha}, \mathrm{Mg}^{\prime \prime b ", ~[N I I] ~}$ & ELG \\
\hline 4 & A & $074651+561042$ & 2002.02 .05 & $0.0353 \pm 0.0005$ & 3600 & $\mathrm{H}_{\alpha}$ & ELG \\
\hline 5 & A & $074732+554148$ & 2002.02.06 & $0.0333 \pm 0.0006$ & 4200 & $\mathrm{H}_{\alpha}$, [SIII], [OIII] & HII \\
\hline 6 & A & $074735+554118$ & 2001.01.16 & $0.0342 \pm 0.0004$ & 3000 & $\mathrm{Mg} “ b ”, \mathrm{NaD}$ & G \\
\hline 7 & A & $074745+554641$ & 2002.02.06 & $0.0332 \pm 0.0005$ & 5100 & $\mathrm{H}_{\alpha},[\mathrm{OIII}],[\mathrm{SII}]$ & HII \\
\hline 8 & $\mathrm{R}$ & $074751+554706$ & 2002.02 .05 & $0.0800 \pm 0.0004$ & 4800 & $\mathrm{H}_{\alpha},[\mathrm{SII}]$ & $\mathrm{S} 2 / \mathrm{L}$ \\
\hline 9 & A & $074755+554628$ & 2001.01.16 & $0.0353 \pm 0.0005$ & 3600 & $\mathrm{H}_{\alpha},[\mathrm{NII}]$ & ELG \\
\hline 10 & A & $074757+554510$ & 2002.02 .05 & $0.0346 \pm 0.0004$ & 3900 & $\mathrm{NaD}, \mathrm{Mg}^{\prime} \mathrm{b} "$ & G \\
\hline 11 & A1 & $074808+554815$ & 2002.02 .06 & $0.0288 \pm 0.0006$ & 4800 & Mg“b” & G \\
\hline 12 & A & $074836+554858$ & 2001.01.17 & $0.0358 \pm 0.0003$ & 1500 & {$[\mathrm{NII}], \mathrm{H}_{\alpha}, \mathrm{NaD}(\mathrm{VV} 9-13-57)$} & ELG \\
\hline 13 & A1 & $074836+554858$ & - & $(0.024)$ & - & DA 240 LVS & (HII) \\
\hline 14 & A & $074844+560035$ & 2002.02.06 & $0.0362 \pm 0.0006$ & 5400 & $\mathrm{NaD} ?$ & $\mathrm{G} ?$ \\
\hline 15 & $\mathrm{R}$ & $074901+552532$ & 2002.02 .04 & $0.0416 \pm 0.0004$ & 4500 & $\mathrm{H}_{\alpha}, \mathrm{H}_{\beta}, \mathrm{NaD}, \mathrm{Mg}^{\prime} \mathrm{b}$ ” & ELG \\
\hline 16 & B & $074909+553616$ & 2001.01.17 & $0.0183 \pm 0.0004$ & 3600 & $\mathrm{H}_{\alpha}, \mathrm{H}_{\beta}, \mathrm{NaD},[\mathrm{NII}],[\mathrm{SII}](\mathrm{SBS} 0745+557)$ & HII \\
\hline 17 & A & $074912+554312$ & 2002.02.04 & $0.0374 \pm 0.0013$ & 3540 & $\mathrm{NaD}, \mathrm{Mg}^{\prime} \mathrm{b} "$ & G \\
\hline 18 & $\mathrm{R}$ & $074912+555233$ & 2002.02.06 & $0.1237 \pm 0.0008$ & 4800 & $\mathrm{H}_{\alpha}, \mathrm{NaD}, \mathrm{Mg}$ "b" & $\mathrm{S} 2 / \mathrm{L}$ \\
\hline 19 & A & $074924+555813$ & 2002.02 .04 & $0.0340 \pm 0.0004$ & 4500 & $\mathrm{H}_{\alpha},[\mathrm{NII}], \mathrm{NaD}$ & $\mathrm{S} 2 / \mathrm{L}$ \\
\hline 20 & A & $074937+555244$ & 2002.02 .04 & $0.0357 \pm 0.0004$ & 4200 & $\mathrm{H}_{\alpha},[\mathrm{SII}],[\mathrm{OIII}]$ & HII \\
\hline 21 & A & $074958+555528$ & 2001.01.17 & $0.0358 \pm 0.0004$ & 3600 & $\mathrm{H}_{\alpha}, \mathrm{H}_{\beta},[\mathrm{NII}],[\mathrm{SII}],[\mathrm{OIII}](\mathrm{VV}$ 9-13-66) & LINER \\
\hline 22 & A & $075007+553514$ & 2001.01 .16 & $0.0334 \pm 0.0004$ & 2700 & $\mathrm{H}_{\alpha}, \mathrm{NaD}, \mathrm{Mg}$ "b", $G$ band & G \\
\hline 23 & B & $075008+552303$ & - & $(0.0193)$ & - & UGC 04035 & - \\
\hline 24 & B & $075016+552429$ & 2001.01.17 & $0.0203 \pm 0.0004$ & 3600 & $\mathrm{H}_{\beta}, \mathrm{Mg}^{\prime} \mathrm{b} ”, \mathrm{NaD}$ (CGCG262-035) & G \\
\hline 25 & A & $075019+554041$ & 2002.02 .05 & $0.0322 \pm 0.0006$ & 3800 & $\mathrm{NaD},[\mathrm{NII}], \mathrm{Ca} ?$ & G \\
\hline 26 & A1 & $075021+555401$ & 2002.02 .04 & $0.0251 \pm 0.0006$ & 3000 & $\mathrm{H}_{\alpha}$, [NII], Mg“b” (CGCG262-036) & ELG \\
\hline 27 & B & $075028+552901$ & 2002.02 .04 & $0.0190 \pm 0.0004$ & 3600 & $\mathrm{H}_{\alpha}, \mathrm{NaD},[\mathrm{NII}], \mathrm{Mg}^{\prime} \mathrm{b} ”$ & $\mathrm{~S} 2 / \mathrm{L}$ \\
\hline 28 & B & $075053+552758$ & 2002.02.04 & $0.0196 \pm 0.0005$ & 3600 & $\mathrm{H}_{\alpha},[\mathrm{NII}],[\mathrm{SII}]$ & HII \\
\hline
\end{tabular}

western counterpart. Although the "hotspots" found in some radio sources are merely small-scale features in their radio jets (Bridle et al. 1994), this does not seem to be the case in DA 240. Tsien (1982) has found that its bright one has the morphology expected of a true hotspot. The reason for the brightness difference is unclear. Although Doppler boosting can be invoked in superluminal sources, this seems implausible here, given the source size, the boosting factor required, and the near equality of distance to the central nucleus. While it might be possible that the eastern lobe is encountering a pocket of high density, there is no hint of this in the polarization behaviour, and the nearest galaxy (in projection) is VV 9-13-66, $\simeq 100 \mathrm{kpc}$ or more distant.

In addition VV 9-13-66 is a radio source, as was apparent from the first high resolution observations of DA 240 (Willis et al. 1974), and we note that several of the other galaxies are also weak radio emitters: No. 19, and either Nos. 5 or 6 , or both. Slee et al. (1998), in a study of radio galaxies in clusters, find that at the equivalent luminosity (just above $10^{22} \mathrm{~W} \mathrm{~Hz}^{-1}$ ), $3 \%$ of galaxies are radio emitters. We would therefore expect
0.3 radio galaxies in this group, while we find ten times this amount. Without more data, one can only speculate as to the cause of the radio emission from these galaxies seen along the lobes of DA 240. One possibility must be that some of the relativistic electrons from DA 240 are captured (and perhaps reaccelerated) in the galaxy magnetospheres, or in the nuclear region. The question could be considered further with high resolution, high sensitivity radio maps.

\subsection{Properties of the DA 240 group}

From published work we know that no X-ray emission has been detected from DA 240, either from the galaxy itself or from its surroundings. The limits to extended emission (corrected for our assumed $\left.H_{0}\right)$ give a luminosity limit $(3 \sigma)$ of $L_{\mathrm{x}}<1.8 \times 10^{42} \mathrm{erg} \mathrm{s}^{-1}$ (Miller et al. 1999). Similarly, no neutral hydrogen has been detected from the galaxy and its surroundings (van Breugel et al. 1983). Both of these findings are consistent with the low density of ionised gas inferred from the radio polarization of the extended emission (discussed in the 


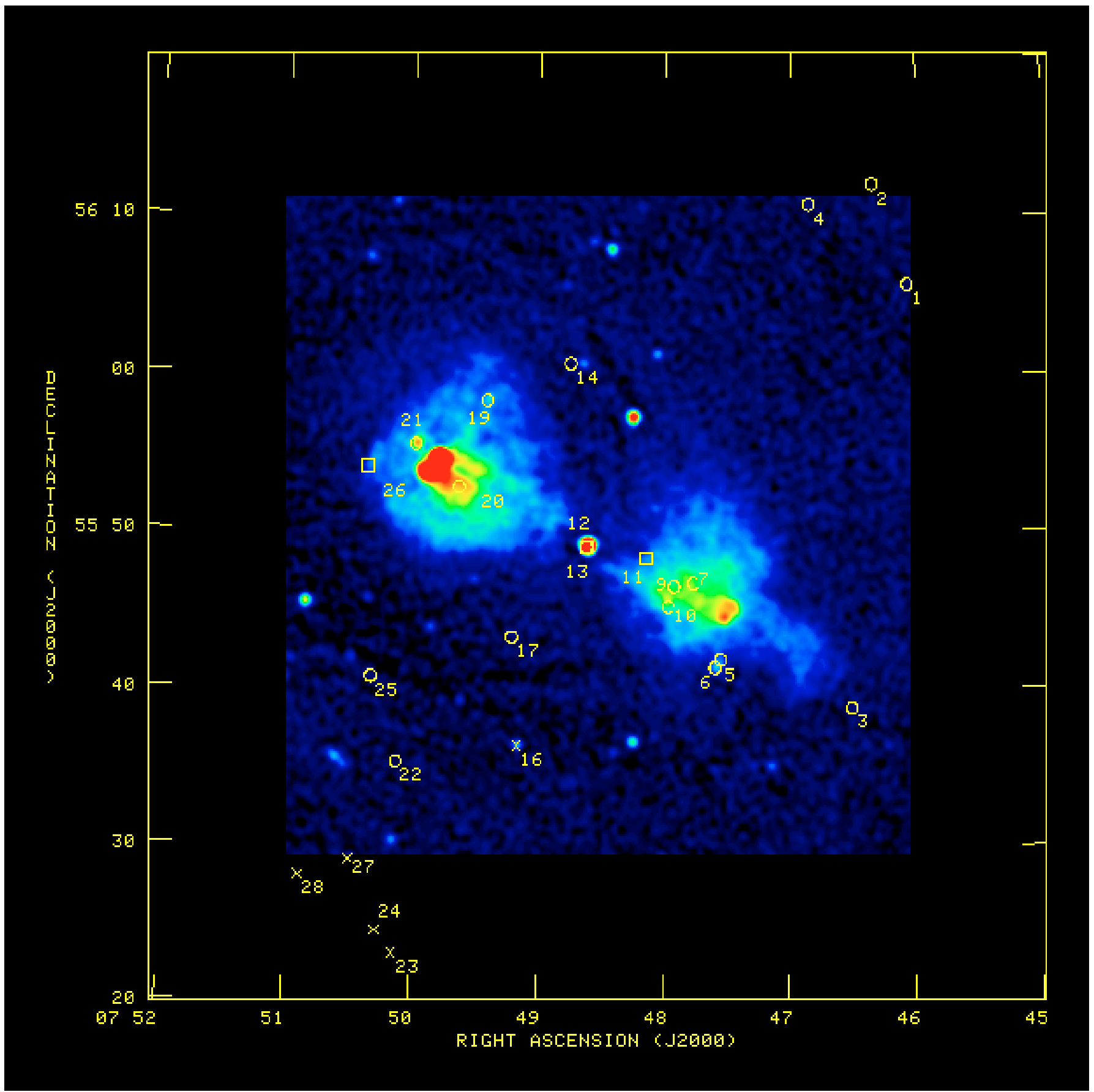

Fig. 4. DA 240 colour map obtained with the WSRT at $49 \mathrm{~cm}$, with optical galaxies of groups A, A1 and B (identified in Table 2) indicated. Group A of 17 galaxies, at redshifts $0.032 \leq z \leq 0.037$, is plotted with circles; group A1 ( $z \simeq 0.025)$ using squares; and group B of 5 galaxies, at $z \simeq 0.019$, with crosses in the map.

previous section). They are also consistent with the observed properties of nearby $(z<0.35)$ FRII radio sources (Wan \& Daly 1996; though as noted above, DA 240 lies on the FRI/II borderline).

What is unusual for nearby FRII sources, however, is for them to be members of a group or cluster. As our observations clearly demonstrate, DA 240 lies in such a group, and is moreover the dominant member of that group. The red magnitudes of the 17 members are shown in Fig. 5, with VV 9-13-57 considerably brighter than the rest ${ }^{1}$. If this is a fair indication of its mass (including dark matter), then it must be the dominant member of the group kinematically as well.

Figure 6 shows the velocity distribution of the 17 group members, in effect the main peak in Fig. 3, with finer binning. As reference point $(v=0)$ we have taken the median velocity

1 As noted in Sect. 2.1, the magnitudes used are on a "stellar scale." The effective scale for galaxies may also not be linear over the magnitude range of our objects. 


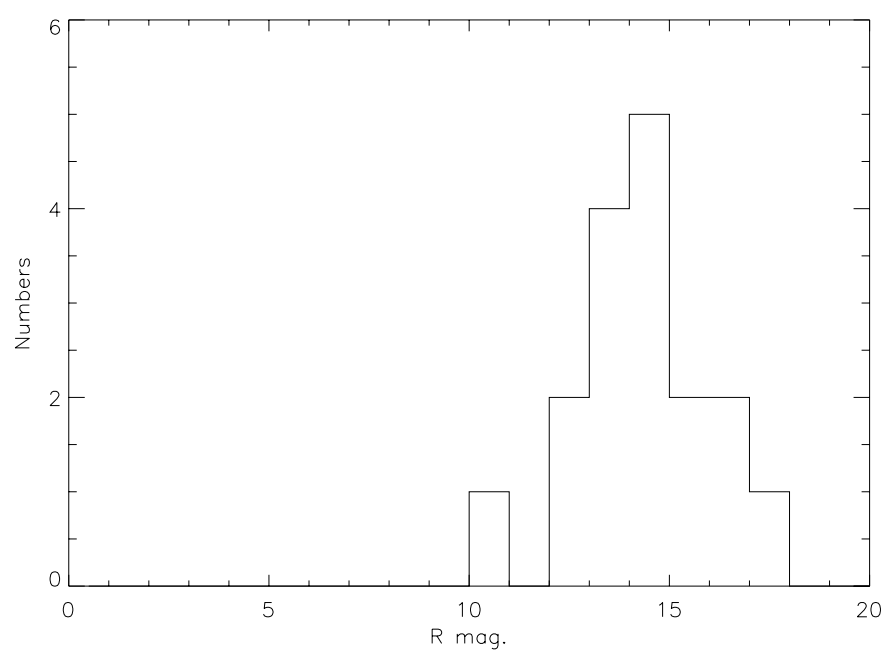

Fig. 5. A histogram showing the red magnitudes of the 17 confirmed members of the DA 240 group.

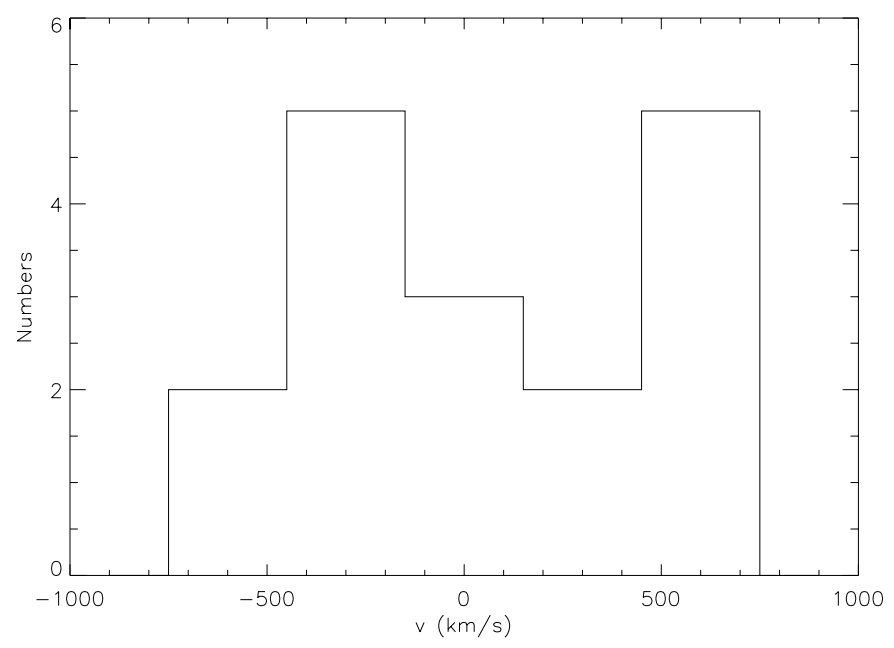

Fig. 6. A histogram of velocity referred to the median for all members of the DA 240 group.

in the distribution (that of galaxy No. 6), though this differs by only $-60 \mathrm{~km} \mathrm{~s}^{-1}$ from the mean. There is a suggestion of bimodality, though given the small number of objects it is probably barely significant. A $\chi^{2}$ test, comparing this distribution with a Gaussian of the same mean $v$ and $\sigma$, indicates that the chance that the velocities are normally distributed is $15 \%$. A bit surprising, perhaps, is that VV 9-13-57 is offset from the median by $+480 \mathrm{~km} \mathrm{~s}^{-1}$.

Let us consider briefly the case of the group A1 sources (with $z \simeq 0.025$ ). Van Breugel et al. (1983) concluded that the LVS (galaxy No. 13) is physically unconnected with VV 9-1357 , and merely seen in projection against it. As clinching evidence, they noted the presence of another galaxy (our No. 11) at nearly the same redshift: the LVS is one member of a foreground group. We confirm the redshift of No. 11, and find moreover a third member, No. 26, at $z=0.025$. This appears to further strengthen the case made by van Breugel et al. (1983), though there is one slightly disturbing fact. All three A1 galaxies are seen in projection against the components of DA 240. For now we conclude that this alignment (which only consists of 3 objects, so its statistical significance is marginal) is merely accidental, and that A1 is a small foreground association, but a search for more members would be desirable.

Turning now to our spectral classifications (Table 2, last column), we note that about $40 \%$ (7/17) are classified as normal ("G"). (Of the other galaxies an even smaller proportion belongs to this category.) However, if we only consider group A galaxies along the radio axis, then the proportion of normal galaxies falls to $20 \%$. Also, as noted above, a number of these have detected radio emission. Though the numbers involved are small, it would appear that there is some degree of enhanced activity among the galaxies linked to the DA 240 radio emission.

\subsection{The unusual association}

The most striking finding is that a group of 10 galaxies is aligned with the two lobes of DA 240. Six other galaxies in the group lie roughly orthogonal to the radio axis. What could the cause of this unusual grouping be?

As we argued above, the chance of the galaxies being aligned with the radio lobes having occurred by accident is quite small. To quantify this, we have done a Monte-Carlo simulation to determine the probability of the observed alignment. The radio lobes were defined by two lines passing through VV 9-13-57, $19^{\circ}$ on either side of the radio source major axis. This region includes 10 galaxies (it excludes No. 14), so we have estimated the probability that 10 or more of them would appear there at random. Locations for the 17 galaxies in the group (Sect. 3.3) were then randomly generated, and this was repeated $10^{7}$ times. This exercise suggests that the configuration observed (Fig. 4) will only occur by chance at a rate of $3 \times 10^{-4}$, implying that a causal association is more likely. Although we did not carry out a systematic survey for such alignments in the first instance, the number of double radio galaxies implicitly "searched" amounts to no more than a few tens; as noted below, one other example has long been known.

If there is a causal relationship, the question is, what is cause and what is effect? Did the location of the galaxies somehow influence where the radio emission would end up, or could something connected with the radio activity somehow have "triggered" galaxy formation? We note that VLBI observations of the nuclear component in DA 240 (Saripalli et al. 1997) define a major axis fairly closely aligned with the outer hotspots. The connection of the galaxy group must therefore be with something in the nucleus of VV 9-13-57, most likely its rotation axis.

To investigate the matter further, we have looked at the kinematics of the galaxies which lie on or near the radio lobes, 11 in total (including VV 9-13-57). We have projected the location of each galaxy onto an axis defined by the radio core and component hotspots of DA 240. Figure 7 shows the relative velocity $\Delta v$ (referred to the median of the entire group, see Sect. 3.3) as a function of distance along the radio axis, $D$. There is a clear correlation of $\Delta v$ with $D$ (correlation coefficient of -0.74): the galaxies are evidently moving at rates which increase with their mutual distances. The gradient is, $\Delta v / D=425 \mathrm{~km} \mathrm{~s}^{-1} \mathrm{Mpc}^{-1}$. Based on the existing data, there 


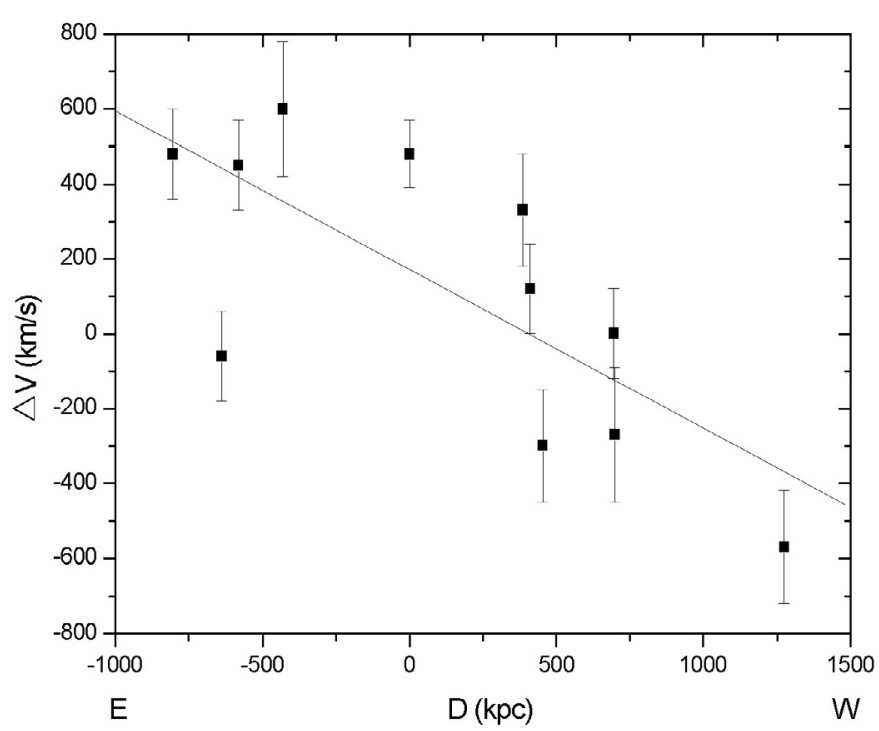

Fig. 7. Relative velocity $(\Delta v)$ of galaxies along the lobes of DA 240 as a function of distance from the host, VV 9-13-57. The line is a least-squares fit to the points.

is no single unique geometry which can explain this result. We will consider two simple configurations which are consistent with it.

Perhaps the simplest explanation is that we are seeing galaxies moving close to the radio axis in a nearly collinear manner. The radio axis is inclined with respect to the plane of the sky, so the line-of-sight velocity is $\neq 0$. Since we do not know the sense of the inclination, the galaxies can either be expanding apart, or contracting inwards. (It might be possible to resolve this ambiguity by determining the inclination of VV 913-57 if it has a dust lane, though this may be complicated by the LVS.) For this discussion we will assume they are expanding. We also do not know the degree of inclination, but the large (projected) size of the radio source suggests it cannot be greatly foreshortened. Assuming the angle to the line of sight is $60^{\circ}$ (the median value for a randomly-oriented ensemble of rods), we can correct the observed $\Delta v / D$ for projection effects and obtain $\Delta v_{\mathrm{c}} / D_{\mathrm{c}}=740 \mathrm{~km} \mathrm{~s}^{-1} \mathrm{Mpc}^{-1}$. Its inverse gives a dynamic timescale for the system, $t_{\mathrm{dyn}}=1.3 \times 10^{9} \mathrm{yr}$. It seems natural to associate this with the time since a major event (intense interaction? explosion?) involving these members of the group.

A rather different explanation is that we are seeing galaxies in a nearly edge-on ring, orbiting something at the centre (presumably the dominant host, VV 9-13-57). The length of the group then corresponds to the diameter of the ring, the velocities at the extrema give the orbital speed. For these we have, $d / 2=r \simeq 1 \mathrm{Mpc}$ and $v_{\text {orb }} \simeq 425 \mathrm{~km} \mathrm{~s}^{-1}$. The enclosed mass is, $M=r v_{\text {orb }}^{2} / G=4.1 \times 10^{13} M_{\odot}$. Although the implied mass is plausible, this configuration seems unlikely for several reasons. The probability of seeing a ring nearly edge on is small. Some explanation is required for why the radio axis should coincide with the plane of the ring; if their alignment is accidental, we have another small probability.

Neither of these simple configurations offers an explanation for the other six galaxies in the group, to the northwest and southeast. One can only assume they were too distant from the centre to have taken part in the antics of their cousins, and leave their orthogonal disposition to happenstance.

Finally, we note that the alignment of galaxies found here, while unusual, is not unique. Another well-studied example involves NGC 383, which is also a very large radio galaxy (3C 31, with a size approaching $1 \mathrm{Mpc}$; Strom et al. 1983) with a chain of companions along the radio source axis. The galaxies in the group have been extensively studied by Sakai et al. (1994), who find 11 members in the chain. Their velocity dispersion $\left(550 \mathrm{~km} \mathrm{~s}^{-1}\right)$ is rather similar to that found for the DA 240 group. In their discussion of the radio emission, Strom et al. note that it is possible that the gravitational attraction of the companions of NGC 383 has influenced the direction of the lobes of 3 C 31 .

\section{Concluding remarks}

Our results conclusively show that there is a group of galaxies around VV 9-13-57, the host of the giant radio source DA 240. Moreover, most of the group members cluster about the major axis of the radio components. The velocity gradient we observe in this subgroup establishes a kinematic timescale near $10^{9} \mathrm{yr}$. If, as seems not unreasonable, the DA 240 host galaxy came to be the dominant member of the group through repeated mergers, then we suggest that the galaxies along the radio axis are "debris" from the last merger episode 1 Gyr year ago. The merging material also provides fuel to supply (and perhaps trigger) nuclear activity. The radio emission now observed has largely been produced in the last 10 million years. That the (low density) radio components should make their way to regions of high galaxy density seems to be counter-intuitive, and we are not yet able to resolve this conundrum. Our investigations of this fascinating group continue.

Acknowledgements. The Westerbork Synthesis Radio Telescope is operated by The Netherlands Foundation for Research in Astronomy (ASTRON), with financial support from The Netherlands Organisation for Scientific Research (NWO). We thank the Chinese Academy of Sciences and the Royal Dutch Academy of Sciences for financial support during visits of RGS to the NAOC which facilitated this project. We are very grateful to Ms. Y. Fan and Dr. C. Jin for helping with some of the data reduction. This project is supported by the NSF of China through grant no. 10173015 and 19973014. BP acknowledges grant NKBRSF 2003CB716703. JW acknowledges grant NKBRSF G19990754. This research has made use of the NASA Astrophysics Data System Abstract Service and of the NASA/IPAC Extragalactic Database (NED) which is operated by the Jet Propulsion Laboratory, Caltech, under contract with the National Aeronautics and Space Administration. We acknowledge use of the USNO-A2.0 catalogue, for which we are most grateful.

We thank an anonymous referee for comments and suggestions.

\section{References}

Baars, J. W. M., Genzel, R., Pauliny-Toth, I. I. K., \& Witzel, A. 1977, A\&A, 61, 99

Baker, J. R., Preuss, E., Whiteoak, J. B., \& Zimmermann, P. 1974, Nature, 252, 552

Barthel, P. D., Schilizzi, R. T., Miley, G. K., Jägers, W. J., \& Strom, R. G. 1985, A\&A, 148, 243 
Bridle, A. H., Davis, M. M., Fomalont, E. B., Willis, A. G., \& Strom, R. G. 1979, ApJ, 228, L9

Bridle, A. H., Hough, D. H., Lonsdale, C. J., Burns, J. O., \& Laing, R. A. 1994, AJ, 108, 766

Falco, E. E., Kurtz, M. J., Geller, M. J., et al. 1999, PASP, 111, 438

Fanaroff, B. L., \& Riley, J. M. 1974, MNRAS, 167, L31

Högbom, J. A., \& Brouw, W. N. 1974, A\&A, 33, 289

Jägers, W. J. 1986, Ph.D. Thesis, University of Leiden

Jägers, W. J. 1987a, A\&AS, 71, 75

Jägers, W. J. 1987b, A\&AS, 71, 603

Klein, U., Mack, K.-H., Strom, R. G., Wielebinski, R., \& Achatz, U. 1994, A\&A, 283, 729

Laing, R., Riley, J. M., \& Longair, M. 1983, MNRAS, 204, 151

Mack, K. H., Klein, U., O’Dea, C. P., \& Willis, A. G. 1997, A\&AS, 123,423

Mack, K. H., Klein, U., O’Dea, C. P., Willis, A. G., \& Saripalli, L. 1998, A\&A, 329, 431

Massey, P., Strobel, K., Barnes, J. V., \& Anderson, E. 1988, ApJ, 328, 315

Miller, N. A., Owen, F. N., Burns, J. O., Ledlow, M. J., \& Voges, W. 1999, AJ, 118, 1988

Noordam, J. E., \& De Bruyn, A. G. 1982, Nature, 299, 597

Rines, K., Geller, M. J., Diaferio, A., Mohr, J. J., \& Wegner, G. A. 2000, AJ, 120, 2338

Sakai, S., Giovanelli, R., \& Wegner, G. 1994, AJ, 108, 33
Saripalli, L., Mack, K.-H., Klein, U., Strom, R. G., \& Singal, A. K. 1996, A\&A, 306, 708

Saripalli, L., Patnaik, A. R., Porcas, R. W., \& Graham, D. A. 1997, A\&A, 328, 78

Schoenmakers, A. P., de Bruyn, A. G., Röttgering, H. J. A., \& van der Laan, H. 2001, A\&A, 374, 861

Slee, O. B., Roy, A. L., \& Andernach, H. 1998, Aust. J. Phys., 51, 971

Stoffel, H., \& Wielebinski, R. 1978, A\&A, 68, 307

Strom, R. G., \& Willis, A. G. 1980, A\&A, 85, 36

Strom, R. G., Baker, J. R., \& Willis, A. G. 1981, A\&A, 100, 220

Strom, R. G., Fanti, R., Parma, P., \& Ekers, R. D. 1983, A\&A, 122, 305

Tsakiris, D., Leahy, J. P., Strom, R. G., \& Barber, C. R. 1996, in IAU Symp., 175, ed. R. D. Ekers, C. Fanti, \& L. Padrielli (Dordrecht: Kluwer Academic Publishers), 256

Tsien, S. C. 1982, MNRAS, 2000, 377

van Breugel, W., Heckman, T., Bridle, A., et al. 1983, ApJ, 275, 61

Veilleux, S., \& Osterbrock, D. E. 1987, ApJS, 63, 295

Wan, L., \& Daly, R. A. 1996, ApJ, 467, 145

Willis, A. G., Strom, R. G., \& Wilson, A. S. 1974, Nature, 250, 625

Willis, A. G., \& Strom, R. G. 1978, A\&A, 62, 375

Willis, A. G., Wilson, A. S., \& Strom, R. G. 1978, A\&A, 66, L1

Yahil, A., \& Vidal, N. 1977, ApJ, 214, 347

Zwicky, F., \& Herzog, E. 1966, Catalogue of Galaxies and Clusters of Galaxies (Pasadena: California Institute of Technology) 